\title{
Isotropic bicelles stabilize the functional form of a small multidrug-resistance pump for NMR structural studies
}

\author{
Sébastien F. Poget, Sean M. Cahill, and Mark E. Girvin \\ Biochemistry Department, Albert Einstein College of Medicine, 1300 Morris Park Avenue, Bronx, \\ NY 10461, USA, girvin@aecom.yu.edu
}

\section{Abstract}

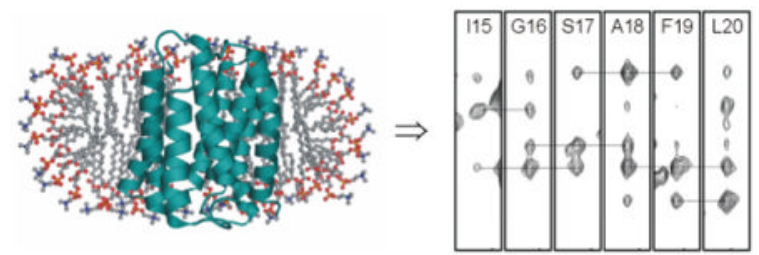

Detergent micelles are commonly used as solubilization agents in biophysical and biochemical studies of membrane proteins, but they do not ideally reproduce the membrane environment and often fail to support the native protein conformation. Bicelles, which are a mixture of short- and longchain lipids, have long been suggested as a more native-like solubilizing agent for the study of membrane proteins. We tested the use of isotropic bicelles as a system for solution NMR studies of membrane proteins on a small multidrug-resistance protein (Smr), a protein that has so far resisted unambiguous structural characterization by X-ray crystallography. We show that the protein can be reconstituted in its functional form and native oligomerization state in bicelles. With an NMR assignment strategy that makes use of sequential NOE information obtained from a NOESY-TROSY and amino-acid specific information from a TROSY-HNCA experiment, $55 \%$ of backbone $\mathrm{H}^{\mathrm{N}}, \mathrm{N}$ and $\mathrm{C}^{\alpha}$ resonances could be assigned, showing that isotropic bicelles are a promising system for NMR structural studies of membrane proteins and are especially suited for the study of a conformationally flexible protein like Smr.

\begin{abstract}
Although detergent micelles are commonly used as membrane mimetics in biophysical studies of membrane proteins, they do not ideally reproduce the native environment of the protein in the membrane and may fail to support the native fold of the protein. Not surprisingly, these non-physiological conformations are particularly common for helical membrane proteins that undergo significant conformational changes as part of their functional cycle, such as the voltage sensor domain of potassium channels, G-protein coupled receptors and bacterial multidrug transporters. One dramatic example is the small multidrug-resistance pump EmrE from Escherichia coli, which was found in three significantly different conformations in two X-ray structures in detergent ${ }^{1 ; 2}$ and a cryo-electron microscopy $3 \mathrm{D}$ reconstruction map from $2 \mathrm{D}$ crystals $^{3}$. The functional relevance of these structures is the subject of current debate ${ }^{4}$, and an alternative method of getting high-resolution structural information about the functional protein is certainly needed. Bicelles, which are a mixture of short- and long-chain lipids, have long been suggested as a more native-like solubilizing agent for the study of membrane proteins ${ }^{5-7}$, and have been used in a number of biophysical and structural studies of these proteins (for a recent review, see ${ }^{8}$ ). Here we show that bicelles are a promising system in the
\end{abstract}


study of Smr, the EmrE homologue from Staphylococcus aureus, since they both preserve the ligand-binding activity and produce NMR spectra that allowed making substantial NMR backbone assignments for this conformationally flexible protein.

Smr ( Staphylococcal multidrug-resistance protein, also called QacC) is a 107 amino acid membrane protein that utilizes the proton gradient across the membrane to pump a range of lipophilic, mostly cationic drugs out of the bacterium in an antiporter manner ${ }^{9}$. It is functional as a dimer of four transmembrane (TM) helix monomers, with the highly conserved Glu 13 residues from both monomers found in close proximity at the dimer interface 10 and involved in both proton and drug binding ${ }^{11}$. Given the highly dynamic nature of small multidrugresistance pumps evident from previous structural studies, the right choice of membrane mimetic becomes essential in order to study a functionally relevant form of the protein ${ }^{4}$.

To compare the abilities of different membrane mimetics to sustain the native conformation of Smr, we tested the protein's ability to bind tetraphenylphosphonium (TPP), a known ligand of $\mathrm{Smr}^{9}$. The protein was purified using an organic solvent extraction and purification scheme $^{12 ; 13}$ and then reconstituted into a number of different detergents and bicelles. The ability of the reconstituted proteins to bind ligand at $\mathrm{pH} 8$ was monitored by fluorescence quenching of the unique native tryptophan 62 , in a fashion similar to a drug binding assay developed for EmrE ${ }^{14}$. Among the tested detergents, reproducible binding was only found for decyl- and dodecylmaltoside (DM and DDM), with only non-specific binding detected in $\beta$ nonylglucoside (NG), the detergent used in the crystal structure determinations of $\mathrm{EmrE}^{1 ; 2}$, as well as in dodecylphosphocholine (DPC), dihexanoylphosphatidylcholine (DHPC), sodium dodecanoylsarcosine, sodium dodecylsulfate (SDS) and lysopalmitoylphosphatidylglycerol (LPPG). In addition, excellent ligand binding properties were observed in dihexanoyl-/ dimyristoylphosphatidylcholine bicelles (biPC) with $q$ values (i.e. long chain to short chain lipid ratio) between 0.25 and 0.5 (see Figure 1). The dissociation constants for the Smr/TPP complex in bicelles, decyl- and dodecylmaltoside were determined to be $3.5,49$ and $3.7 \mu \mathrm{M}$, respectively. The $\mathrm{pH}$ dependence of TPP binding was also tested for both DDM and biPC, and as previously reported for EmrE ${ }^{11}$ and expected for this proton antiporter, only non-specific binding was observed at $\mathrm{pH} 6.0$ (data not shown). In addition, equilibrium sedimentation analysis 15 was used to show that the protein is present in its native dimeric oligomerization state in bicelles (Figure S1 in the Supporting Information) and to obtain an estimate of $150 \pm$ $50 \mathrm{kDa}$ for the protein/bicelle complex size.

NMR spectra of Smr were collected in both biPC $q 0.33$ and decylmaltoside at $\mathrm{pH}$ 6.5. In both conditions, ${ }^{1} \mathrm{H}^{15} \mathrm{~N}$-TROSY spectra ${ }^{16}$ of comparable quality were obtained, and the overall similarity in peak positions indicated that the protein was present in essentially the same conformation in both systems. However, at $\mathrm{pH} 8.0$ where drug binding should be observable, no spectra could be obtained in DM due to a very rapid degradation in signal intensities, whereas samples in biPC still yielded reasonable quality TROSY spectra. We therefore decided to ascertain whether further studies by solution NMR would be possible in bicelles by obtaining NMR resonance assignments for this system.

One of the main problems for the NMR experiments on samples of Smr in bicelles is the short sample lifetime due to protein precipitation. In order to alleviate this, we optimized the bicelle composition by using the ether-linked (non-hydrolyzable) DMPC and DHPC analogues and spiking the bicelles with $10 \%$ of negatively charged dihexanoyl-/ dimyristoylphosphatidylserine in the same $3: 1$ ratio ${ }^{17-19}$. These improved samples retained bicelle-like character as shown by their ${ }^{31} \mathrm{P}-\mathrm{NMR}$ spectra 6 and displayed sample half-life times of 10-14 days at $\mathrm{pH} 6.5$ and $42{ }^{\circ} \mathrm{C}$. We also confirmed that Smr was still able to bind TPP in these bicelles using the fluorescence binding assay. 
Due to the large size of the protein/bicelle complex, most triple-resonance experiments conventionally used for making assignments yielded only a small subset of cross-peaks mostly from the termini and the loop regions of the protein. Therefore, the assignment strategy that we employed was based on two experiments: a 3D NOESY-TROSY experiment ${ }^{20}$ (collected on an $800 \mathrm{MHz}$ spectrometer with cryoprobe) to obtain sequential amide-to-amide NOEs and a 3D TROSY-HNCA experiment ${ }^{21}$ (collected on a $900 \mathrm{MHz}$ spectrometer with cryoprobe) to obtain $\mathrm{C}^{\alpha}$ chemical shifts as well as some additional sequential information. Analysis of these two datasets allowed the unambiguous assignment of $55 \%$ of amide and $\mathrm{C}^{\alpha}$ atoms (Figure 2, Figure S2). The assignments are dispersed throughout the protein sequence, covering most of TM1 (including the drug- and proton-binding residue Glu 13) and parts of TM2, TM3 and TM4, with the longest stretch of unassigned sequence being 19 amino acids long (see Table S1). The main problem in assigning the remaining observable TROSY peaks was in the ambiguity of sequential NOEs and the lack of inter-residue information from the TROSYHNCA experiments for those peaks. A strategy for obtaining full assignments will therefore involve the preparation of specifically amino-acid labeled samples to identify a number of anchor points in the unassigned regions of the protein and thus resolve ambiguous sequential NOEs.

Even with the current assignments, it will be possible to study the ligand binding sites for different drugs at atomic level in order to gain valuable insight into the drug binding and transport mechanism. After additional assignments, distance constraints from paramagnetic relaxation effects in spin-labeled samples and residual dipolar coupling restraints will be used to define the structure of Smr in this very membrane-like environment. Because only one crosspeak per residue is found in the spectra, the present data suggest that functionally competent Smr exists as a symmetric dimer in bicelles, unlike the asymmetric conformations found in the $\mathrm{X}$-ray crystal structures.

Our work represents the first example where substantial solution NMR assignments for a polytopic membrane protein in isotropic bicelles have been obtained and shows that small bicelles are a viable and promising system for the study of a highly dynamic $8 \mathrm{TM}$ protein by NMR. Together with the current progress in the use of magnetically aligned bicelles (see the recent structure of the $2 \mathrm{TM}$ protein MerFt by Opella and co-workers ${ }^{22}$ ), these methods hold great promise in reducing the bottleneck of membrane protein structure determination. Finally, because bicelles more closely mimic the membrane environment, they should be suitable for the study of a large proportion of membrane proteins, therefore reducing the need for timeconsuming detergent screens for every protein to be studied.

\section{Supplementary Material}

Refer to Web version on PubMed Central for supplementary material.

\section{Acknowledgements}

We would like to thank Dr. S. Khrapunov for helpful discussions, Drs. S. C. Almo and M. D. Brenowitz for instrument use and the New York Structural Biology Center for instrument time and support. Financial support was received from the NIH (NYSBC: GM66354; MG: GM72085) and from the Swiss National Science Foundation and the Swiss Foundation for medical-biological Grants (SFP).

\section{References}

1. Ma C, Chang G. Proc Natl Acad Sci USA 2004;101:2852-2857. [PubMed: 14970332]

2. Pornillos O, Chen YJ, Chen AP, Chang G. Science 2005;310:1950-1953. [PubMed: 16373573]

3. Ubarretxena-Belandia I, Baldwin JM, Schuldiner S, Tate CG. EMBO J 2003;22:6175-6181. [PubMed: 14633977] 
4. Tate CG. Curr Opin Struct Biol 2006;16:457-464. [PubMed: 16828280]

5. Sanders CR, Schwonek JP. Biochemistry 1992;31:8898-8905. [PubMed: 1390677]

6. Glover KJ, Whiles JA, Wu G, Yu N, Deems R, Struppe JO, Stark RE, Komives EA, Vold RR. Biophys J 2001;81:2163-2171. [PubMed: 11566787]

7. Vold RR, Prosser RS, Deese AJ. J Biomol NMR 1997;9:329-335. [PubMed: 9229505]

8. Prosser RS, Evanics F, Kitevski JL, Abdul-Wahid MS. Biochemistry 2006;45:8453-8465. [PubMed: 16834319]

9. Grinius LL, Goldberg EB. J of Biol Chem 1994;269:29998-30004. [PubMed: 7962000]

10. Koteiche HA, Reeves MD, Mchaourab HS. Biochemistry 2003;42:6099-6105. [PubMed: 12755611]

11. Muth TR, Schuldiner S. EMBO J 2000;19:234-240. [PubMed: 10637227]

12. Yerushalmi H, Lebendiker M, Schuldiner S. J Biol Chem 1995;270:6856-6863. [PubMed: 7896833]

13. Krueger-Koplin RD, Sorgen PL, Krueger-Koplin ST, Rivera-Torres AO, Cahill SM, Hicks DB, Grinius L, Krulwich TA, Girvin ME. J Biomol NMR 2004;28:43-57. [PubMed: 14739638]

14. Elbaz Y, Tayer N, Steinfels E, Steiner-Mordoch S, Schuldiner S. Biochemistry 2005;44:7369-7377. [PubMed: 15882076]

15. Reynolds JA, Tanford C. Proc Natl Acad Sci USA 1976;73:4467-4470. [PubMed: 188041]

16. Pervushin K, Riek R, Wider G, Wuthrich K. Proc Natl Acad Sci USA 1997;94:12366-12371. [PubMed: 9356455]

17. Ottiger M, Bax A. J Biomol NMR 1999;13:187-191. [PubMed: 10070759]

18. Struppe J, Whiles JA, Vold RR. Biophys J 2000;78:281-289. [PubMed: 10620292]

19. Triba MN, Zoonens M, Popot JL, Devaux PF, Warschawski DE. Eur Biophys J 2006;35:268-275. [PubMed: 16187128]

20. Zhu G, Kong XM, Sze KH. J Biomol NMR 1999;13:77-81.

21. Salzmann M, Pervushin K, Wider G, Senn H, Wuthrich K. Proc Natl Acad Sci USA 1998;95:1358513590. [PubMed: 9811843]

22. De Angelis AA, Howell SC, Nevzorov AA, Opella SJ. J Am Chem Soc 2006;128 UR:12256-12267. [PubMed: 16967977] 


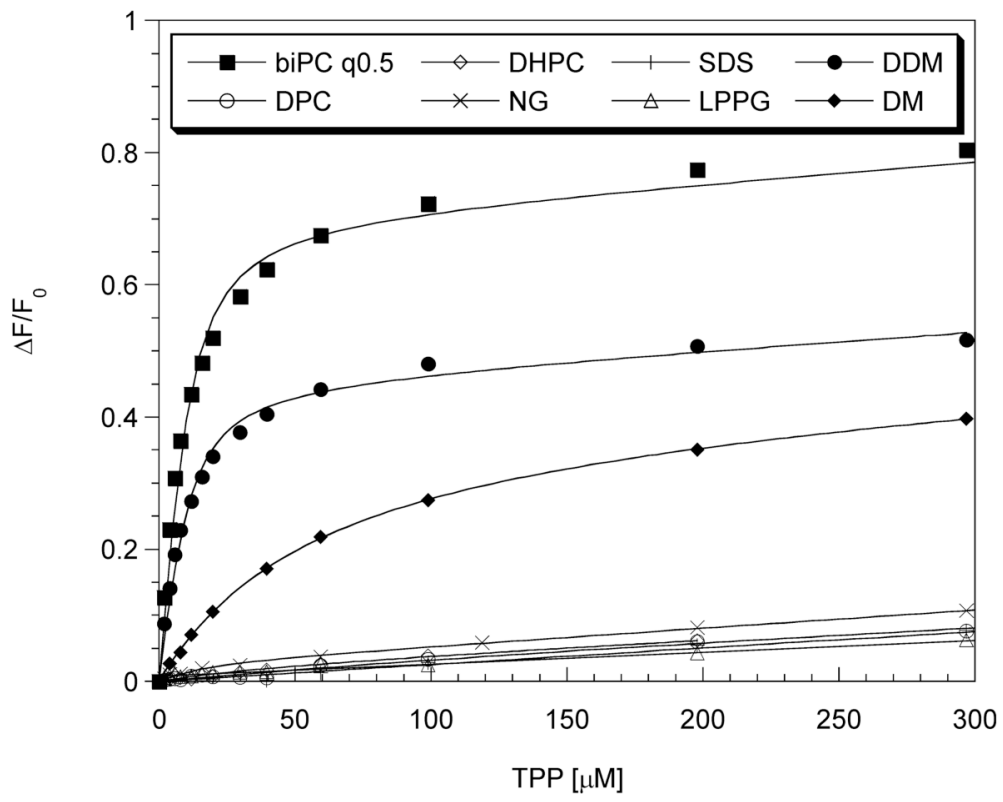

Figure 1.

TPP binding to Smr reconstituted in different detergent and bicelle systems at $\mathrm{pH}$ 8.0. The relative fluorescence change upon addition of TPP is plotted and the solid lines represent the best fit of the binding function to the data. 


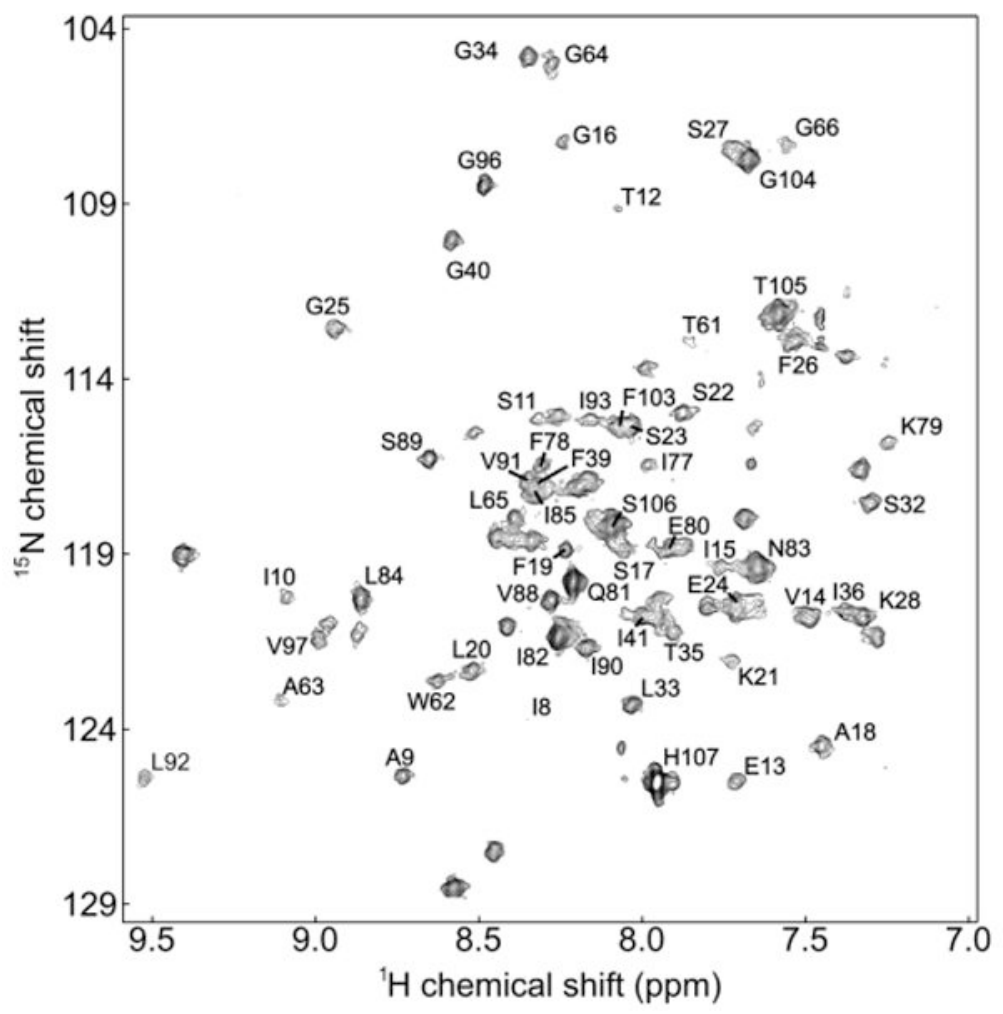

Figure 2.

The $900 \mathrm{MHz}{ }^{1} \mathrm{H}^{15} \mathrm{~N}$-TROSY spectrum of $0.8 \mathrm{mM}$ uniformly ${ }^{2} \mathrm{H},{ }^{13} \mathrm{C},{ }^{15} \mathrm{~N}$-labeled Smr in etherlinked, PS-spiked $q 0.33$ bicelles at $\mathrm{pH} 6.5$ and $47^{\circ} \mathrm{C} ; 256$ increments with 64 scans each were acquired in the ${ }^{15} \mathrm{~N}$-dimension. More than $90 \%$ of the cross-peaks expected for a symmetric dimer are present, and assigned peaks are labeled. 